\title{
Gross Dissection of Liver for Hepatocellular Carcinoma Using AJCC Cancer Staging Manual 8th Edition: Anatomical and Practical Considerations
}

\author{
Marla Beach ${ }^{1} \bullet$ Laura Henao Caviedes ${ }^{1}$ - Consolato M. Sergi \\ ${ }^{1}$ Department of Laboratory Medicine and Pathology, University of Alberta Hospital and \\ Stollery Children's Hospital, Edmonton, AB, Canada; ${ }^{2}$ Departments of Pediatrics, \\ Laboratory Medicine and Pathology, Stollery Children's Hospital, University of Alberta, \\ Edmonton, AB, Canada.
}

Author for Correspondence: Marla Beach, Department of Laboratory Medicine and Pathology, University of Alberta Hospital and Stollery Children's Hospital, Edmonton, AB, Canada. Email: Marla.Beach@albertaprecisionlabs.ca

Doi: https://doi.org/10.36255/exonpublications.livercancer.2021.ch4

\begin{abstract}
Hepatocellular carcinoma is one of the most common causes of cancerrelated death worldwide. In cases of liver resection for hepatocellular carcinoma, the dissector, whether a pathologist's assistant, physician, or resident, must have a clear understanding of both the terminology of liver anatomy and the requirements of the corresponding College of American Pathologists Cancer Protocol to properly orient, describe, dissect, and sample the specimen. This chapter provides guidance for the gross dissection procedure for the production of a valuable pathology report, which is of key importance for a patients' ongoing care.
\end{abstract}

Keywords: AJCC cancer staging manual; Couinaud segments; gross dissection of liver; hepatocellular carcinoma; pathology

In: Liver Cancer. Sergi CM. (Editor). Exon Publications, Brisbane, Australia. ISBN: 978-0-6450017-2-3; Doi: https://doi.org/10.36255/exonpublications.livercancer.2021

Copyright: The Authors.

License: This open access article is licenced under Creative Commons Attribution-NonCommercial 4.0 International (CC BY-NC 4.0) https://creativecommons.org/licenses/by-nc/4.0/ 


\section{INTRODUCTION}

The liver is a solid, asymmetrical organ normally located in the right upper quadrant of the abdomen and is found exclusively in vertebrates (1). An exception to this rule occurs in the case of heterotaxis or Ivemark Syndrome, in which the liver is located centrally or to the left side of the body (2). The liver synthesizes proteins and produces biochemicals, which are specifically important for digestion and growth. The gallbladder is a small sac, which is found on the visceral side of the liver. The gallbladder is adherent to the posterior liver along one side (the gallbladder 'bed'), and in rare cases, is not visible as it can be entirely intrahepatic (3). A thorough inspection should be given in cases of biliary atresia with Kasai porto-enterostomy, because the gallbladder can be very small or absent (4). The gallbladder stores and delivers bile produced by the liver into the digestive tube.

The commonly used College of American Pathologists (CAP) Cancer Protocols detail protocol for reporting, designed to produce a standardized, comprehensive reporting of hepatocellular carcinoma (5). These synoptics are derived from the AJCC Cancer Staging Manual ( $8^{\text {th }}$ ed) (6). The main categories of a complete pathological report, using the CAP synoptic, employs three main categories: (i) primary tumor (T stage), (ii) regional lymph nodes ( $\mathrm{N}$ stage), and (iii) distant metastasis (M stage). As with most pathological specimens, unless a metastasectomy is performed, gross bench considerations are primarily $\mathrm{T}$ and $\mathrm{N}$ staging. Therefore, the M stage is not discussed here.

\section{GROSS AND SURGICAL ANATOMY OF THE LIVER}

Gross descriptors of liver anatomy, in practice, are generally an ad hoc miscellany of older anatomical landmarks (7), along with the more functional Couinaud liver segments (8). Generating and reviewing gross descriptions of liver resections require an understanding of both.

\section{Surface inspection}

Surface inspection of the liver reveals many anatomical landmarks. These include the falciform ligament, the ligamentum teres (round ligament), porta hepatis, gallbladder, and the inferior vena cava (or its groove). Porta hepatis, or the hilum, contains the veins entering the liver (portal vein), the hepatic artery, and the bile ducts exiting the liver. The falciform ligament is thin, membranous, and attaches the liver to the anterior abdominal wall. At the liver's inferior edge, the falciform ligament merges with the round ligament (the umbilical vein's remanent). The round ligament sits in a grove called the umbilical fissure. Macroscopically, the Falciform ligament has commonly been used as the border between the right and left lobes. It grossly sits at a junction where the liver is larger (right lobe) and smaller (left lobe). This is the anatomical right and left lobes. If inspected from below (surgeon's point of view) or posteriorly on the dissection table, four additional 'lobes' are appreciated. At the top, just medial to the IVC groove is a square to rectangular protrusion designated the caudate lobe (caudate is Latin for 'tail', 
as if it were the tail of the liver). Below the caudate lobe is the gallbladder (centre right), and the hilum (centre left). The gallbladder is a soft sac, commonly green or purple, adjacent to the posterior and inferior liver. The hilum in the resected liver will likely have multiple sutures, clips, and staples on its structures: portal vein and hepatic artery entering the liver, and the bile ducts exiting the liver. The round ligament sits to the left of the hilum and delineates the left anatomical lobe on the posterior liver surface. The quadrate lobe is not a distinct lobar structure, rather, it is a four-sided area (hence 'quad') bounded by the porta hepatis superiorly, the gallbladder laterally, the round ligament medially, and the free edge of the liver inferiorly.

Three main hepatic veins drain from the liver superiorly; the right, middle and left hepatic veins. In the resected total liver, careful inspection of the IVC or its groove will reveal the left and central hepatic veins. Commonly, adjacent to the IVC to the right, the right hepatic vein is found.

\section{Couinaud segments}

Further understanding of blood and bile flow in the liver informs the functional description of liver segments described by Couinaud (8). This system separates the liver, based on studies done with corrosion casting into eight independent segments. The middle hepatic vein level, as it extends from inferior to drain into the IVC superiorly, divides the functional right and left liver in this system. At the gross level, an estimation of this border is Cantlie's line. Cantlie's line is an imaginary line through the fossa of the inferior vena cava to the gallbladder's fossa, extending through to the front of the liver (9). Each segment has its own biliary drainage and vascular inflow and outflow. In the centre of each segment, there are branches of the portal vein, hepatic artery, and bile duct. In the periphery of each liver subsegment, there is a vascular outflow through the hepatic veins. The main dividing lines of liver segments in the transverse plane are the right and left portal veins. Dividing lines into the vertical and oblique planes are the three main hepatic veins (right, middle, and left). The segments are numbered clockwise from 1-8 (or I-VIII) (Figure 1). In this system, Segment 1 corresponds to the caudate lobe. Segments 2-4 comprise the functional right lobe (to the right of Cantlie's line), and segments 5-8 comprise the functional left lobe (to the left of Cantlie's line). The only segment that is often subdivided is segment 4 , into segments 4 a (superior) and $4 \mathrm{~b}$ (inferior). The quadrate lobe does not have a corresponding functional segment, rather is part of segment $4 \mathrm{~b}$. The Falciform ligament does not divide the functional lobes, but the anatomical lobes; Cantlie's line better approximates functional division of right and left lobes and is the line between the right lobe segments 5, 6, 7 and 8, and the left lobe segments 2, 3 and 4 .

\section{Adjacent organs and their impressions on the liver}

There are several impressions on the surface of the liver, accommodating the adjacent organs. These neighboring organs are key because they can be part of the resection in cases where the adjacent organs are adherent and possibly involved by invasive tumor. A classic example is a hepatocellular carcinoma directly and contiguously invading the right colonic flexure. Underneath the right lobe, and to 

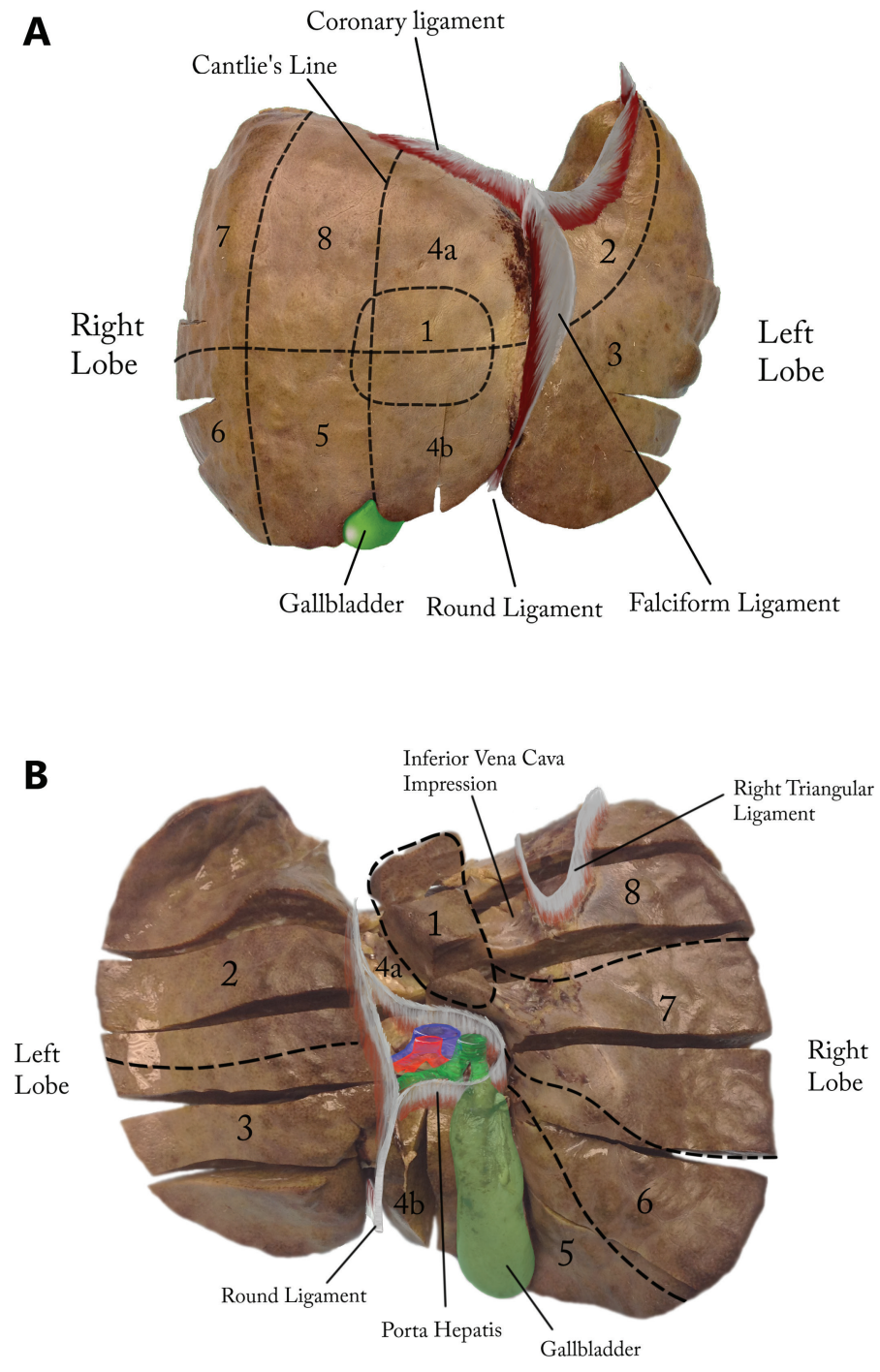

Figure 1. Functional (Couinaud) segments of the liver. A. Anterior surface. B. Posterior surface. The segments 1-8 are delineated by hashed lines in relation to the anatomical landmarks.

the right of the gallbladder for some, it is essential to recognize two impressions which are one behind the other and separated by a ridge. The first is the colic impression, a shallow liver parenchyma compression that is formed by the hepatic flexure of the large bowel. The second is the renal impression, which lies behind the colic impression. It accommodates part of the right kidney and part of the suprarenal gland. Other important impressions include the duodenal and gastric impressions. The duodenal impression has a distinct, characteristic shape and can be found medial to the renal impression and lies between the segment 8 and the 
neck of the gallbladder. Visible on the left lobe of the liver, on the inferior surface is the gastric impression. Lastly, while not an impression, rather a point of contact, is the superior, rounded surface of the right and mid liver, where tumour involvement of the diaphragm is possible.

\section{Grossing}

When a surgical procedure is performed to resect a liver mass, the specific procedure needs to be correctly identified. It is important to separate total hepatectomy from partial hepatectomy and wedge resection. Total hepatectomies are received in the setting of orthotopic liver transplantation (10). Thorough review of clinical history will enlighten the dissector as to the surgeon's intraoperative findings, the lobes, segments, any structures removed, as well has any history of previous liver surgeries, diagnoses, or therapies. Particular attention should be paid to the surgical report, number, location, and size of previously identified tumors, as well as previous therapies such as resection, and oncological or ablative procedures (11). The partial hepatectomy is subdivided into: (i) major hepatectomy, when three segments or more are involved, and (ii) minor hepatectomy, when the resection involves only one or two segments. Review the clinical information regarding the type of resection, anatomical lobe, and the segments that were resected. Wedge resections are small and may have one, two, or three sides with the identifiable liver capsule.

\section{Preparation of fresh tissue}

All liver resections should be prepared in the fresh state to aid in immediate and complete formalin fixation. Tissue should be weighed and measured in three dimensions. If a gallbladder is present, this should also be measured. Inking should be performed. Inking serves two purposes: to mark surgical resection margin(s) and mark and aid assessment of areas of concern. The surgical resection margin is where the liver parenchyma was transected by the surgeon and is often cauterized and may have many surgical staples or sutures. Other areas that can be inked are roughened areas where the liver was dissected away from other organs or possible tumor involvement locations. Roughened anatomical areas may include 'bare' areas where the liver was in contact with other organs, or the gallbladder bed, for example. While there are normally some roughened areas of the liver capsule these may also indicate where a tumor may involve or perforates the liver capsule. As such these can be inked in various colors so that they can be easily found and assessed after cutting. After weighing, measuring, and inking, the tissue is serially sectioned and submerged in formalin for fixation.

\section{Gross inspection, description, and sampling}

At the gross bench, one proceeds with a structured approach to any specimen (12). This ensures all findings are captured, and cases are consistent. One common approach is the outside to inside approach, which is a systematic inspection and description of an organ from the outer surface (capsule or serosa) to its cut surface's inside structures and appearance. For the liver, we inspect the outer surface, 
identify the size, shape, landmarks, and attached structures to determine what was resected. It is imperative to liaise with the surgeon under challenging cases to ensure the specimen's proper handling.

\section{Tumor}

With regard to the tumor, it is crucial to identify all tumors. The largest five masses must be described in detail. When multiple tumors are present, care must be taken not to confuse satellitosis with multiple primary tumors. A satellite nodule is smaller than the main tumor and will sit within two centimeters from it, separated by background liver tissue. All tumors must be described with regards to their size, location (and structures involved), distance to resection margins, cut surface/consistency, and presence of hemorrhage or necrosis. For tumor size, ensure the size is described, three dimensionally, for the largest 5 tumors, in centimeters.

Regarding the tumor location, it is important to specify which functional segment the tumor is found. The functional segments cannot be discerned in the solid organ with the naked eye, hence the use of anatomical landmarks to help approximate segments. Figures $1 \mathrm{~A}$ and $1 \mathrm{~B}$ demonstrate the approximate functional segments overlaid with the gross appearance and anatomical landmarks on a total hepatectomy. With lesions that are at, near, or straddle the areas where two segments meet, it is not uncommon to include both segment numbers in the description. For example, if a mass is identified on the anterior surface, just to the left of the Falciform ligament may be arising from segment 2 or 3 and can be described as located in segment $2 / 3$. Carefully inspect the edges of the tumor(s) for the involvement of vascular structures and select sections to confirm vascular invasion. With regards to the tumor extension, it is important to select all events that apply, including no evidence of primary tumor, tumor confined to the liver, tumor involving a major branch of the portal vein or the involvement of the hepatic veins, the involvement of the visceral peritoneum with obvious perforation, the invasion of the gallbladder, and invasion of the diaphragm, that is, the direct extension of the tumor into other neighboring organs. If the tumor categories cannot be assessed, this also needs to be specified in the protocol.

Margins are those areas of the specimen where the surgeon has dissected, either sharp or blunt, to sever it from the patient. It is essential to recognize the parenchymal margin, which can be involved by the neoplasm. If the neoplasm does not involve the parenchymal margin, the invasive carcinoma distance from the margin in centimeters needs to be stated in the gross description. Additional margins need to be specified if they are required; for example, if there is an attached organ involved by the mass, the distance to the margin(s) of that tissue needs to be recorded.

The cut surface of the mass should be described. This includes color, consistency, and description of the tumor border. Clinical history of ablative, chemotherapeutic or similar treatments is important to note. In these cases, the tumor may be completely or partially necrotic. Inspect the entire tumor and estimate the percent necrosis. Necrosis in treated liver tumors often appears as homogenous, crumbly, soft to firm but friable cut surface mottled green/brown. The surrounding parenchyma is often white, rubbery and fibrous. If residual tumor is present, give the size of residual tumor in three dimensions, in centimeters. 


\section{Lymph nodes}

If resected with part of the hilum, the specimen may include soft tissue, including a few lymph nodes. Ensure all soft tissue at the hilum is carefully inspected, and all potential nodes found are submitted for histology. If the gallbladder is present, also inspect the soft tissue around the cystic duct for any lymph nodes. Other regional lymph nodes of liver tumors (hepatoduodenal ligament nodes, inferior phrenic, and caval nodes) may be received separately. The number of submitted lymph nodes need to be adequately identified. The number of lymph nodes involved, and the number of lymph nodes examined need to be specified in the gross description report.

\section{Pathologic staging}

With regards to the pathologic stage classification according to the pTNM, AJCC $8^{\text {th }}$ edition (6), three descriptors are considered, including the letter " $m$ " as multiple primary tumors, the letter " $r$ " as recurrent, and the letter " $y$ " as posttreatment. In the TNM, a solitary tumour of $2 \mathrm{~cm}$ or less is classified as pT1, while a tumor with $2 \mathrm{~cm}$ or more without vascular invasion is considered pTlb. A solitary tumor of $2 \mathrm{~cm}$ or less is pTla. The labeling of pTl is also used if the tumor size is more than $2 \mathrm{~cm}$ without vascular invasion. The labeling of pT2 regards a solitary tumor of a diameter of more than $2 \mathrm{~cm}$ with vascular invasion or multiple tumors, but none more than $5 \mathrm{~cm}$. In the case of multiple tumors in which at least one is more than $5 \mathrm{~cm}$, the labeling of pT3 is used. Finally, pT4 is used when a single tumor or multiple tumors of any diameter involve a major branch of the portal vein or hepatic vein, or when tumor(s) with specific direct invasion of adjacent organs other than the gallbladder or with perforation of visceral peritoneum are found. The labeling of $\mathrm{pNl}$ is used in case of regional lymph node metastasis, while $\mathrm{pNO}$ when no regional lymph node metastases are seen. The labeling of $\mathrm{pMl}$ is used when distant metastasis is seen and is required only if confirmed pathologically. Additional pathologic findings include fibrosis, cirrhosis, lowgrade dysplastic nodule, high-grade dysplastic nodule, steatohepatitis, iron overload or chronic hepatitis. Also report on fibrosis present and indicate the assessment scale used. The protocol described above applies only to hepatic resection specimens containing hepatocellular carcinoma, including fibrolamellar carcinoma. Carcinoma of the intrahepatic bile ducts are staged using a separate TNM system. This protocol should not be used for combined hepatocellular carcinoma - cholangiocellular carcinoma, sarcomas, and metastatic tumors.

\section{Sampling for histology}

In preparing the cassettes, sections should be prepared from each major tumor nodule with representative sampling of smaller nodules. The locations and pathologic parameters should be provided for the five largest tumors. In the case of cirrhotic nodules that are larger than the surrounding background level, they need to be separately sampled, because such nodules may contain dysplastic changes. Satellite nodules, multifocal primary hepatocellular carcinoma and intrahepatic metastasis are obviously assessed as multiple tumors. In the case radiofrequency ablation or transarterial chemoembolization has been used, the extent of 
necrosis seen grossly and microscopically is crucial, because it needs it to be correlated with the downstaging used on imaging. The tumor needs to be studied microscopically in its entirety when it is up to 2 centimeters in size. In the case of larger tumors, an additional section for each one centimeter is recommended and should be provided to the pathologist. Further sampling is also necessary from the periphery of the tumor or areas that seem to be viable. Overall, a combination of gross and microscopic findings determines the extent of necrosis and should be reported in up to five of the largest tumor nodules. The definition of satellitosis is not uniform, and no universal definition has been provided. It is improper to label as satellite any tumor nodule within a vascular structure, which needs to be better categorized as lymphatic a vascular invasion.

\section{CONCLUSION}

Gross dissection of liver resection specimens for hepatocellular carcinoma is an integral part of a comprehensive, complete pathology report. A systematic approach to these specimens is key to the proper collection of data elements needed for the completion of the relevant CAP cancer protocol synoptic report. Understanding liver anatomy, both traditional anatomical landmarks, and functional liver segments is crucial to translate and correlate gross and clinical findings to ensure an accurate and reliable pathology report that enables the patients' ongoing care.

Conflict of Interest: The authors declare no potential conflicts of interest with respect to research, authorship and/or publication of this chapter.

Copyright and Permission Statement: The authors confirm that the materials included in this chapter do not violate copyright laws. Where relevant, appropriate permissions have been obtained from the original copyright holder(s), and all original sources have been appropriately acknowledged or referenced.

\section{REFERENCES}

1. Polampelli A. What is the liver pathophysiology? J Liver Dis Transplant 2020;9(3):172.

2. Hrusca A, Rachisan AL, Lucian B, Oprita S, Manole S, Cainap S. Ivemark syndrome-a rare entity with specific anatomical features. Rev Med Chil. 2015;143(3):383-6. https://doi.org/10.4067/ S0034-98872015000300014

3. Monib S, Mahapatra P, Fayez H. Intrahepatic Gallbladder. Eur J Case Rep Intern Med. 2019;6(6). https://doi.org/10.12890/2019_001123

4. Yamataka,A. Laparoscopic Kasai portoenterostomy for biliary atresia, J Hepatobiliary Pancreat Sci. 2013;20:481-6. https://doi.org/10.1007/s00534-013-0607-1

5. Protocol for the Examination of Specimens From Patients With Hepatocellular Carcinoma. https:// documents.cap.org/protocols/cp-gihepatobiliary-hepatocellular-20-4100.pdf [Accessed on 11 Feb 2021]

6. Amin MB, Edge S, Greene F, Byrd DR, Brookland RK, Washington MK, et al. AJCC Cancer Staging Manual (8th edition). Springer International Publishing: American Joint Commission on Cancer; 2017. 
7. Bismuth, H. Surgical anatomy and anatomical surgery of the liver. World J. Surg. 1982;6:3-9. https:// doi.org/10.1007/BF01656368

8. Strasberg SM. Nomenclature of hepatic anatomy and resections: a review of the Brisbane 2000 system. J Hepatobiliary Pancreat Surg. 2005;12:351-5. https://doi.org/10.1007/s00534-005-0999-7

9. Cantlie, J. On a new arrangement of the right and left lobes of the liver. Proceedings - Anatomical Society of Great Britain and Ireland. 1897;32:4-9.

10. Makowka L, Stieber AC, Sher L, et al. Surgical technique of orthotopic liver transplantation. Gastroenterol Clin North Am. 1988;17(1):33-51.

11. Weledji EP, Ngounou E. The Impact of Segmental Anatomy on Hepatic Oncologic Resections. Curr Surg Rep. 2016;4(4). https://doi.org/10.1007/s40137-015-0122-1

12. Dayton AS, Ro JY, Schwartz MR, Ayala AG, Raymond AK. Raymond's Paragraph System: an alternative format for the organization of gross pathology reports and its implementation in an academic teaching hospital. Arch Pathol Lab Med. 2009;133(2):298-302. https://doi.org/10.5858/133.2.298 
